\title{
CHARACTERIZATION AND DESIGN OF DIGITIZING PROCESSES FOR UNIFORM AND CONTROLLABLE DROPLET VOLUME IN EWOD DIGITAL MICROFLUIDICS
}

\author{
Jian Gong and Chang-Jin "CJ" Kim \\ Mechanical and Aerospace Engineering Department, University of California, Los Angeles (UCLA) \\ Los Angeles, CA, USA
}

\begin{abstract}
We report characterization and precise control of droplet volume for digital microfluidics under electrowetting-on-dielectric (EWOD) actuation both in air and oil environments. By using multi-layer printed circuit board (PCB) as an EWOD chip and custom-making an automatic control board, the restrictions on the electrode pattern design and driving signal are eliminated, empowering us to run various electrode sequences and electric signals. Reading droplet volumes optically or electrically, we characterize and optimize the droplet creation and cutting processes. The results lead to more uniform and repeatable droplet volume, which is essential for a precision instrumentation application. The tunable droplet volume is also available by changing the digitizing parameters.
\end{abstract}

\section{INTRODUCTION}

Digital microfluidics fulfill fluidic operations by manipulating droplets through various mechanisms such as thermal, chemical, surface wave, dielectrophoresis (DEP), or electrowetting-ondielectric (EWOD), the last currently being the most prominent. In digital microfluidics, droplet volume uniformity and accuracy (digital unit) are two essential factors for many applications such as drug discovery or quantitative analysis. Although the digitizing processes (e.g., creating droplets from bulk liquid) are already included in the device design, the results are further affected by the liquid dynamics. Externally controlled pumps and valves are usually used for droplet dispersion in micro-channel or parallelplate configuration [1][2], which requires extra setup and compromises the simplicity digital microfluidics can bring about for the final systems.

Using EWOD [3], which allows for local modulation of surface wettablity through embedded electrodes, the process of droplet creation, moving, mixing and cutting has been studied in [4]. The droplet creation integrated into the microfluidic chip free of external setup has significantly simplified the system development and device package [5]. The next question is how accurate and precise the volume of such droplets can be. Since the droplet digitization on EWOD chip is affected by details of operation procedures and parameters as well as electrode sizes and shapes, a systematic study has been difficult. Helped by the recent development of a multi-layer EWOD chip and a custom-built electrical control board, which make parametric studies practical, we report the first results of such a study.

\section{EWOD CHIP AND EXPERIMENT SETUP}

Recently we have developed a new EWOD chip fabrication technology by taking advantage of the multi-layer printed circuit board (PCB) that allows direct and independent electrical access to each electrode [6], as shown in Fig. 1. This multilayer electrical connection eliminates the limitation on electrode pattern design and the side connection wires which disturb the droplet digitizing process in the single electrode layer EWOD chip [5].

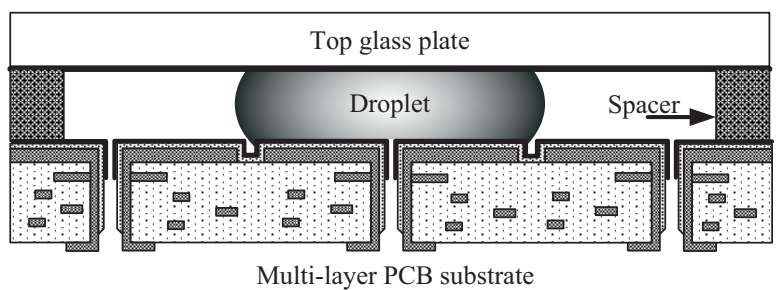

Figure 1. Direct-referencing EWOD chip fabricated with fourlayer printed circuit board (PCB) technology [6].

In Fig. 1, the EWOD chip is made of a PCB substrate and a glass plate. A 4-layer PCB is lapped, polished, and then $8000 \AA$ Parylene $C$ is deposited as dielectric layer and $2000 \AA$ Teflon $\AA$ as hydrophobic coating. The top glass is coated with $2000 \AA$ transparent ITO as a ground electrode and $2000 \AA$ Teflon ${ }^{\circledR}$ as hydrophobic coating. Droplets are then sandwiched between the PCB and the glass by spacers. The device is either exposed to air or filled with oil. In this paper, the EWOD chip has $1.5 \mathrm{~mm} \times 1.5$ mm electrode pads and a $100 \mu \mathrm{m}$-thick spacer between the parallel plates.

Our experiments are performed using the setup shown in Fig. 2 , which measures the droplet volume on chip and controls the EWOD driving voltages by high voltage relay array. Different droplet digitizing processes are tested, and the optical images and electrical signals of the droplet footprint are recorded.

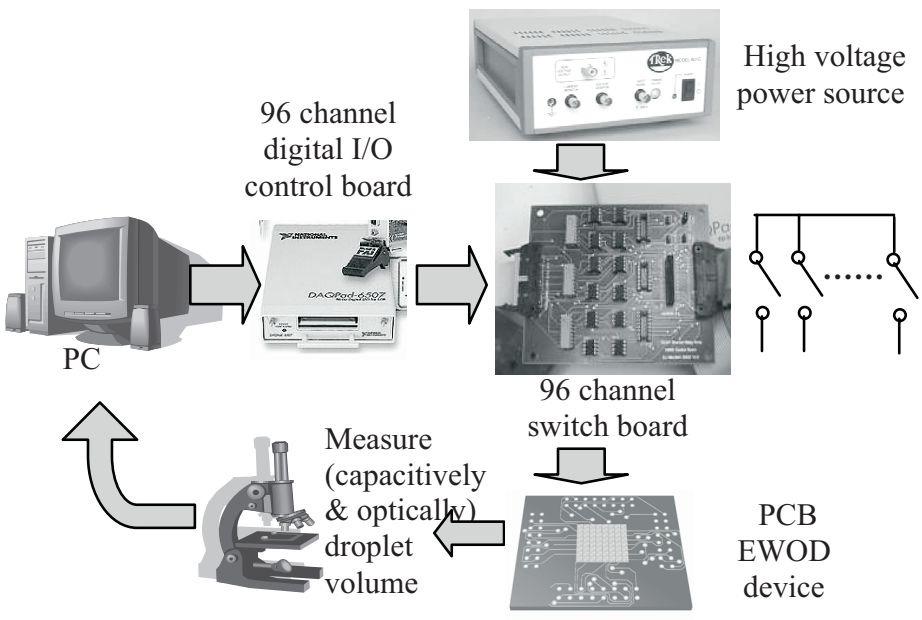

Figure 2. Experiment setup to measure the droplet volume on chip and control the driving signals.

To provide designed electrical signal sequences to the EWOD device, we have custom-built a dedicated automatic electrical control board. In this control board, one 96-channel relay switch 
board is controlled by a National Instruments (NI) DAQPAD-6507 96-channel digital I/O control board. We also developed control program in the upstream $\mathrm{PC}$, which can design, store and send the high voltage switch sequence to the I/O board through a USB port. The resulting control board can switch the high voltage up to 500 $\mathrm{V}$ and simultaneously control the 96 channels as fast as $1 \mathrm{~ms}$.

The droplet volume is calculated by multiplying the measured droplet footprint area with the spacer size. Since the electrodes are much larger than the spacer in the current EWOD chip (15:1) and liquid contact angle is typically near $90^{\circ}$, the error is expected to be less than $1 \%$. We measure the droplet footprint by two different methods. One is by capturing droplet optical images and having their areas read and calculated by Scion Image ${ }^{\circledR}$. We also determine the footprint electrically by measuring the capacitance between the electrodes on the top and bottom plates [5]. Because the droplets are quite flat, i.e., the contribution of the fringe-field is relatively small, a linear relationship is expected between the capacitance and the footprint area. Fig. 3 shows the measured capacitance vs. the optically-measured droplet size. A linear relationship is confirmed, and the calibration between the capacitance and droplet volume can be obtained.

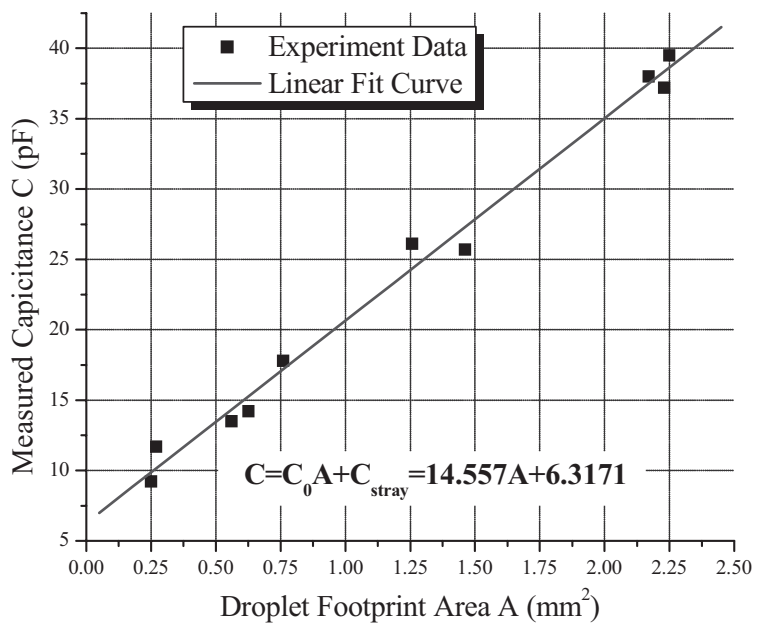

Figure 3. Capacitance measurement vs. droplet footprint area.

\section{EXPERIMENTAL RESULTS}

For droplet creation, the typical method and electrode pattern are shown in Fig. 4 as Creation Procedure I. In this configuration, we design one big electrode ( $9 \mathrm{x}$ bigger than normal size) as the liquid reservoir and two normal size electrodes for pulling and creation sites. To create one droplet from the reservoir, the liquid is first pulled out by the pulling electrode. A droplet is created by activating both the reservoir and creation electrodes but not the pulling electrode. For each step, the turned-on electrodes are activated with $80 \mathrm{~V}_{\mathrm{AC} @ 1 \mathrm{kHz}}$ for $500 \mathrm{~ms}$. Using this method, we can create droplets until the reservoir has less than one droplet volume left. The reservoir is initially filled $70-80 \%$ to ensure enough space for the liquid to be pulled back during the first creation. Overall, 56 droplets can be generated without replenishing during one creation cycle. The results of Creation Procedure I are summarized in Fig. 5, which shows 25 creations over 5 cycles with overall volume variation of $\pm 5 \%$. It is worth noting that the variation was several times worse when the creation procedure was operated manually. The electronic operation was possible only after the custom-built control board became available. Fig. 5 also shows that the volume of generated droplets increases as the liquid left in the reservoir decreases. This is most probably due to the dynamic droplet necking involving competition between two liquid movements at the same time. Because the remaining liquid volume in the reservoir becomes smaller and the resulting pulling back force from the reservoir decreases, more liquid would drift into the creation electrode, generating a bigger droplet.

\section{Creation Procedure I:}
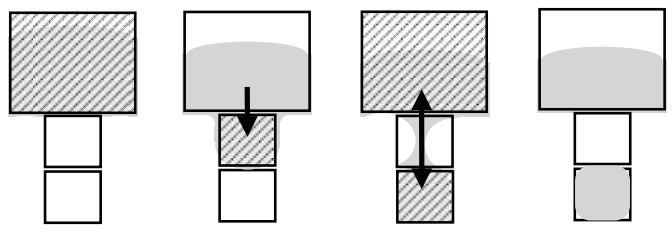

Creation Procedure II (with pre-filling step):
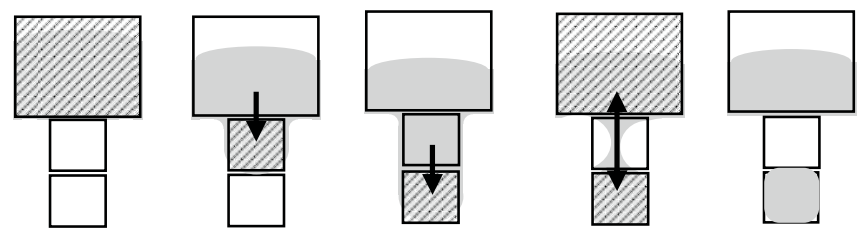

Pre-fill into

creation site

Ground electrode

Activated electrode

Liquid

Figure 4. Two different types of droplet creation procedures. Arrows show the droplet movement direction.

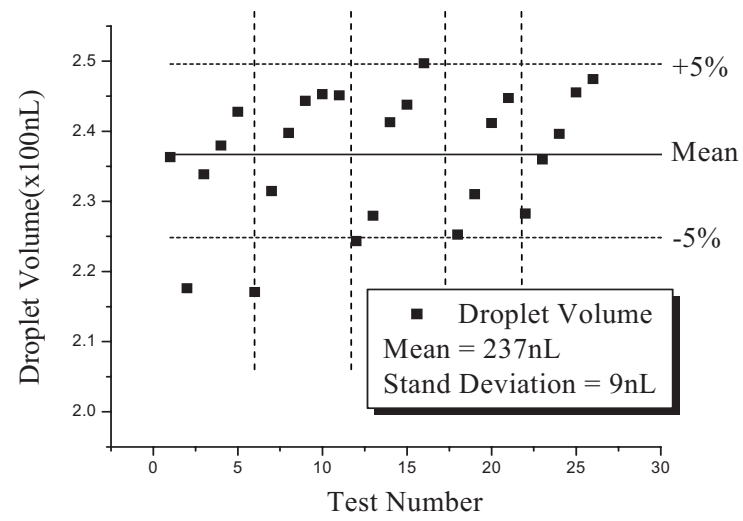

Figure 5. Droplet volume distribution for Creation Procedure I at $80 V_{A C @ 1 k H z}$. Dotted vertical lines separate different creation cycles. Droplet volume increases within a cycle as creation repeats.

To reduce the dynamic competition between the two movements, we designed the Creation Procedure-II shown in Fig. 4, which adds an extra step of pre-filling the creation electrode. Since the creation electrode is pre-filled, during droplet necking, there's just one main liquid movement - pulling back by the reservoir. The liquid already in the creation electrode is held there and its volume is maintained during the droplet necking. Then the droplet volume in the creation site is well defined, and the dynamic effect is minimized. The droplet volume is only determined by where the neck would break. As shown in Figs. 6 and 7(a), the droplet volume distribution by Creation Procedure II is more uniform $( \pm 2 \%)$ than that by Creation Procedure I $( \pm 5 \%)$ and not affected by the volume of liquid remaining in the reservoir. Creation Procedure II was also tested in silicone oil $\left(5 \mathrm{cs}\right.$, Clearco ${ }^{\circledR}$ product), which is another common environmental fluid for EWOD digital microfluidics. Water droplets immersed in oil have much smaller contact angle hysteresis than those in air. Smaller 
hysteresis means the contact lines move with less resistance and so do the droplets. The small resistance makes the EWOD actuation easier, allowing for lower driving voltage, although it in turn reduces the stability of droplets (e.g., against gravity and disturbances). The use of oil also raises such concerns as oil contamination and packaging but helps preventing evaporation of water droplets. Requiring only $30 \mathrm{~V}$ in oil (vs. $80 \mathrm{~V}$ for in-air operation), Creation Procedure II produced droplets with increased volume, as shown in Fig. 7(b).

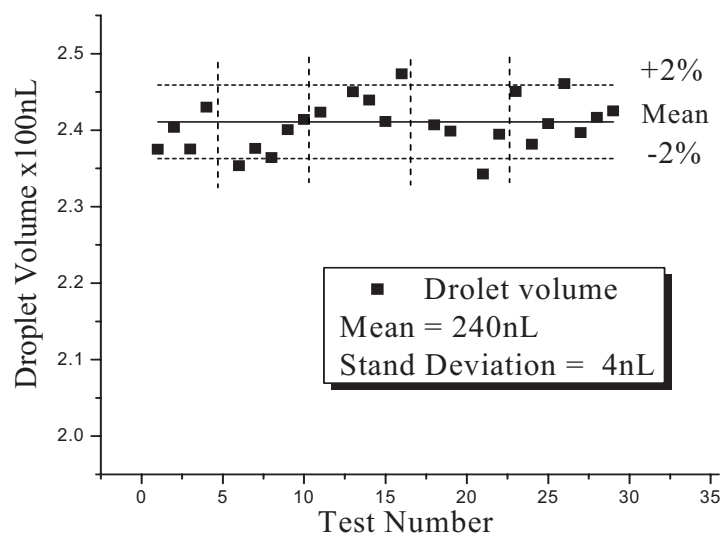

Figure 6. Droplet volume distribution during Creation Procedure II with $80 V_{A C @ I k H z}$. Dotted vertical lines separate different creation cycles (24 creations over 5 cycles). There is no apparent trend of droplet volume changes within one cycle.

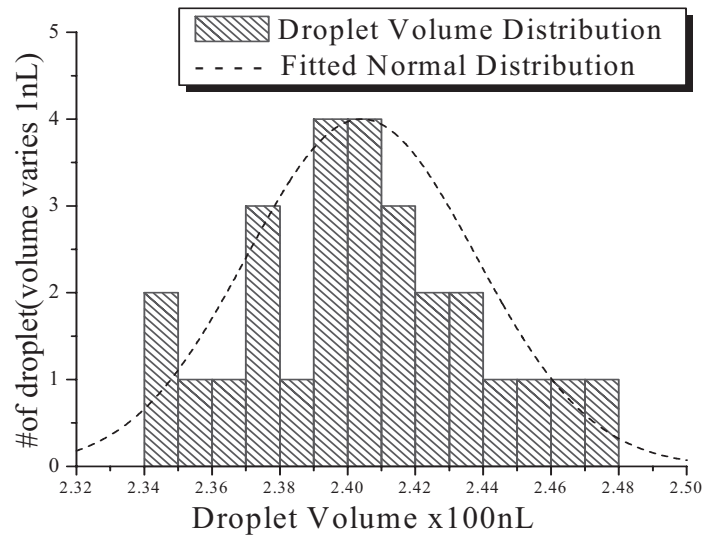

(a) In air. Creation voltage of $80 \mathrm{~V}_{\mathrm{AC} @ 1 \mathrm{kHz}}$ was used.

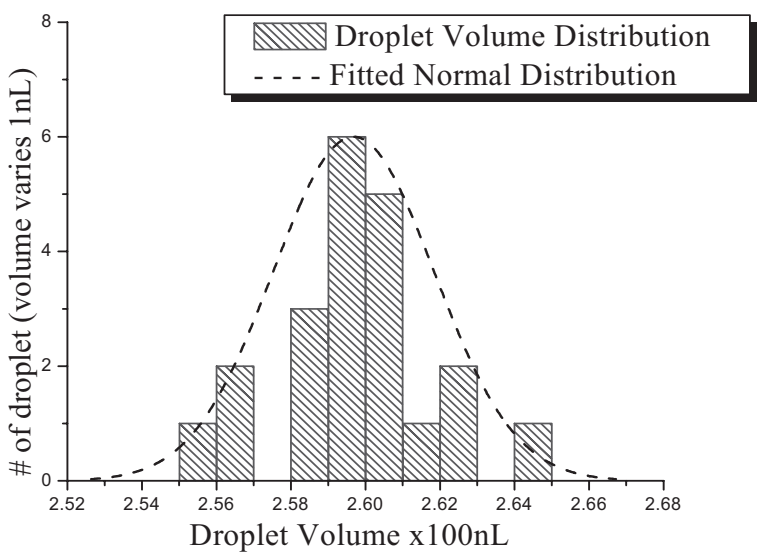

(b) In oil. Creation voltage of $30 \mathrm{~V}_{\mathrm{AC} @ 1 \mathrm{kHz}}$ was used.

Figure 7. Volume distribution of droplets made by Creation Procedure II on EWOD PCB device in air and oil.
The ability to tune the droplet volume is also desirable for a more powerful system. As noticed in the experiments, droplet volume changes with various signal parameters such as driving voltage and signal duration. Using Creation Procedure II, we conducted a series of experiments by varying the voltage applied to creation site. As shown in Fig. 8, we can control the droplet volume from $225 \mathrm{~nL}$ to $245 \mathrm{~nL}(10 \%)$ by changing the creation voltage from $70 \mathrm{~V}$ to $95 \mathrm{~V}$ with $\pm 2 \%$ accuracy at every voltage level.

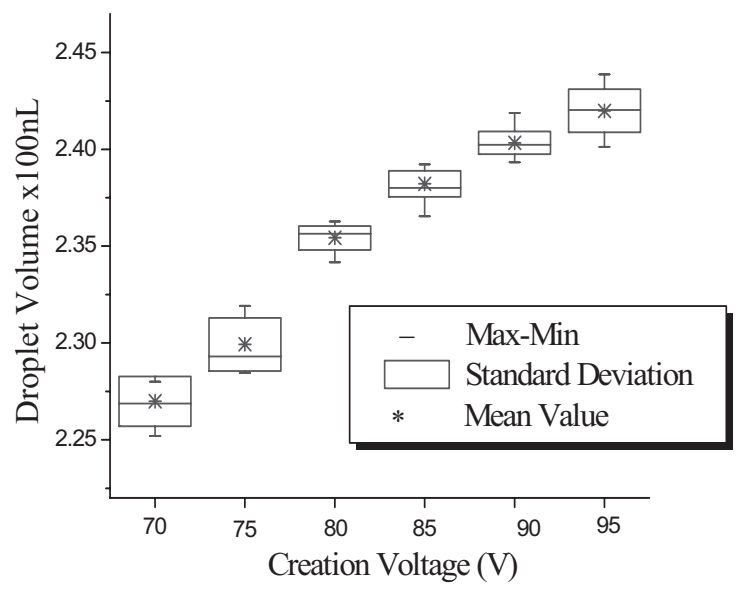

Figure 8. Volume of droplets made by Creation Procedure II for varying voltages (1 $\mathrm{kHz} R \mathrm{RS}$ value).

Dividing a big droplet into two of exactly the same size is more difficult since any asymmetric condition before or during the cutting would lead to an uneven division. As shown in Fig. 9(a) as an example, when a big droplet moves from right to left, its tail would cover more adjacent electrode than its head. If we apply voltage to the two adjacent electrodes on the path of droplet movement to divide the droplet, more liquid would stay in the tail side during the droplet necking, causing the new droplet on the tail side to be bigger than the one on the head side. We may apply a short pulse voltage to the head side electrode to pull the droplet toward the middle, but consistent results are not expected without automatic feedback control. Realizing that the symmetry is maintained perpendicular to the direction of droplet moving, a solution is to cut the droplet perpendicularly, as shown in Fig. 9(b). As shown in Fig. 10, size distribution of the cut droplets is also uniform $( \pm 2 \%)$, and the average cutting ratio (1:0.988) is close to $1: 1$.
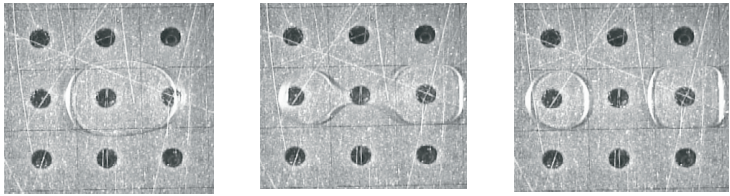

(a) Droplet cutting with asymmetric initial position.
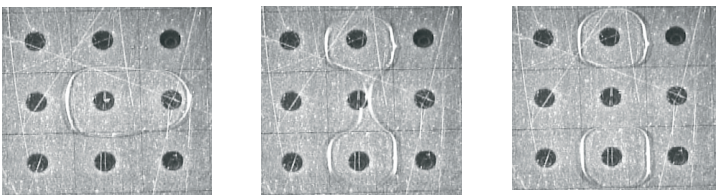

(b) Droplet cutting with symmetric initial position.

Figure 9. Droplet cutting affected by the initial position. Droplet initially moves from right to left. 


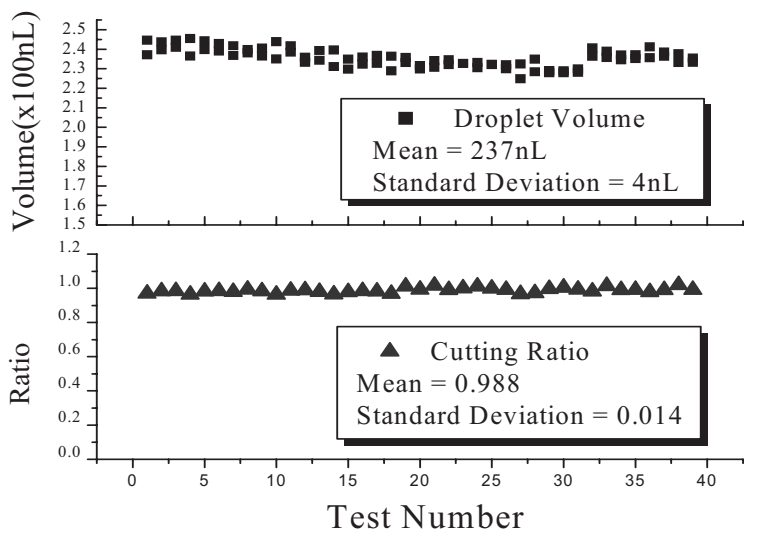

Figure 10. Droplet volume and cutting ratio in the even (1:1) droplet cutting process with $80 V_{A C @ I k H z}$.

\section{DISCUSSIONS}

As we have observed and shown in this paper, droplet creation and cutting are very sensitive processes affected by many factors. By precisely controlled signals and properly designed digitizing procedures, we have alleviated the uncertainties including dynamic effect and improved the uniformity and repeatability of droplet volumes. Although we can control the electrical signal well, the variation among fabricated chips and environmental conditions during operation are unavoidable, such as surface hydrophobicity deteriorating over a long cycle. In a well-controlled lab environment, we can continue monitoring the device performance and tuning the driving signals to compensate for the variations, such as tuning the droplet volume by changing the creation voltage. This means feedback control is desirable for fully automatic digital microfluidics systems. Fast electronic reading of droplet volume is essential for closed-loop feedback control systems, for which the capacitive measurement confirmed in this paper is a good candidate. Currently, the capacitive measurement of droplet volume is being added to the control system board and different feedback algorithms are being investigated to achieve more uniform and tunable droplet volume for digital microfluidics system.

\section{CONCLUSIONS}

We have designed and characterized droplet digitizing processes using the PCB EWOD chip and dedicated electrical control board. Uniform droplet volume distribution $( \pm 2 \%)$ has been achieved for both creation and cutting by a properly designed digitizing procedure and precise electrical control. Tunable droplet volume by changing a control signal parameter suggests that droplet volumes may be prescribed in digital microfluidics system with real-time feedback control.

\section{ACKNOWLEDGEMENT}

This work was supported by the NASA Institute for Cell Mimetic Space Exploration (CMISE) the Jonsson Comprehensive Cancer Center both at UCLA.

\section{REFERENCES}

[1] H. Ren, V. Srinivasan, and R. B. Fair, "Design and Testing of an Interpolating Mixing Architecture for Electrowetting-Based Droplet On-Chip Chemical Dilution," Technical Digest of the $12^{\text {th }}$ International Conference on Solid-Sate Sensors, Actuators and Microsystems (Transducers '03), Boston, USA, (2003), pp. 619622.

[2] Q. $\mathrm{Xu}$ and M. Nakajima, "The generation of highly monodisperse droplets through the breakup of hydrodynamically focused microthread in a microfluidic device", Apply Physics Letter, Vol 85, No.17, pp 3726-3728, (2004).

[3] J. Lee, H. Moon, J. Fowler, C.-J. Kim, and T. Schoellhammer, "Addressable Micro Liquid Handling by Electric Control of Surface Tension", Technical Digest of the $14^{\text {th }}$ International Conference on Micro Electro Mechanical Systems (MEMS '01), Interlaken, Switzerland, (2001), pp. 499-502.

[4] S.K. Cho, H. Moon, and C.-J. Kim, "Creating, Transporting, Cutting, and Merging Liquid Droplets by Electrowetting-Based Actuation for Digital Microfluidic Circuits", Journal of Microelectromechanical. System., Vol. 12, No. 1, pp. 70-80, (2003).

[5] J. Gong, S.-K. Fan, and C.-J. Kim, "Portable Digital Microfluidics Platform with Active but Disposable Lab-On-Chip", Technical Digest of the $17^{\text {th }}$ International Conference on Micro Electro Mechanical Systems (MEMS '04), Masstricht, The Netherlands, (2004), pp. 355-358.

[6] J. Gong and C.-J. Kim, "Two-Dimensional Digital Microfludic System by Multi-Layer Printed Circuit Board", Technical Digest of the $18^{\text {th }}$ International Conference on Micro Electro Mechanical Systems (MEMS '05), Miami, USA, (2005), pp. 726-729. 